\title{
Anti-Reflection and Self-Cleaning Nanocoating to Improve the Performance of Flat Plate Solar Collector
}

\author{
Ahmed M. Ajeena, Piroska Víg, and Istvan Farkas
}

\section{ABSTRACT}

The flat plate solar collector (FPSC) is a well-known energy harvesting tool that minimizes the strain on the traditional energy production approach while also promoting the future use of renewable energy. Thus, Increasing the performance of this collector is worth the effort. As the world's energy consumption continues to grow, there has been an increased focus on the potential for nanotechnology improvements to reduce energy usage. Recent scientific research has shown that the application of nanotechnology can increase the efficiency of the traditional glass cover of FPSC. Antireflective and self-cleaning nanocoating has recently attracted a lot of attention for both its important aspects and wide practical applications. In the current study, there are many sections to the review as follows: (a) general overview of the fundamental theoretical frameworks of antireflection and selfcleaning technology; (b) detailed fabrication methods for both antireflection and self-cleaning coating; (c) A range of anti-reflection coating materials used to add new functionalities for glass cover of FPSC; (d) present the literature of the studies of anti-reflection and selfcleaning coating application in FPSC.

Keywords: Flat plate solar collector, nanocoating, antireflection coating, self-cleaning coating.

Published Online: February 10, 2022

ISSN: 2736-5506

DOI : $10.24018 /$ ejenergy.2022.2.1.41

\author{
A. M. Ajeena* \\ Doctoral School of Mechanical \\ Engineering, Hungarian University of \\ Agriculture and Life Sciences, Godollo, \\ Hungary. \\ Department of Mechanical Engineering, \\ College of Engineering, University of Kufa, \\ Iraq. \\ (e-mail: ajeena.ahmed.mohsin.dhayea@ \\ phd.uni-mate.hu) \\ P. Víg \\ Institute of Mathematics and Basic Science, \\ Hungarian University of Agriculture and \\ Life Sciences, Godollo, Hungary. \\ (e-mail: vig.piroska@uni-mate.hu) \\ I. Farkas \\ Institute of Technology, Hungarian \\ University of Agriculture and Life \\ Sciences, Godollo, Hungary. \\ (e-mail: farkas.istvan@uni-mate.hu)
}

*Corresponding Author

\section{INTRODUCTION}

Increasing a country's social and economic well-being signifies improving its energy production, efficiency, and savings. Because of the new political and economic perspectives on global sustainable development that have emerged over the last decade, a wide range of renewable energy technologies have been developed. A flat plate solar collector (FPSC) isn't an exception to this rule. As a result of its low cost and ease of use, this technology is widely adopted around the world. FPSC has become an important part of both residential and commercial applications. anti-reflection and self-cleaning nanocoating used in solar collectors have been shown to increase photothermal conversion efficiency [1].

Nanotechnology has been used in a wide range of industries, including energy, food, medicine, cosmetics, cars, electronics, textile building, and construction. It is a useful force for the solar system, providing a wide range of resources to deal with energy-related issues. Apply nanotechnology in solar systems such as solar collectors, solar storage, photovoltaic, fuel cell, and dye-sensitive solar cells. Scientists provided extensive research and experiments on solar collector. They worked on the size, shape, angle of radiation, and much more. Nanotechnology is, in fact working on two principal aspects: quantum containment and surface-to-volume ratio. These two aspects are mainly the subject of all new inventions of nanotechnology. Nanotechnology is one of the best methods to improve the efficiency of solar collectors using nanocoating [2].

Nanocoating, also known as nanoparticle coatings, essentially results in applying a one-phase solid structure of which one dimension is lower than $100 \mathrm{~nm}$ on a substrate. Nanocoating are among the essential topics in the field of nanotechnology. These coatings possible have coating built up from layers or interpenetrating by particles less than 100 nanometers, which offer enhanced surface properties. It can be said that any coating with a "nano" attribute, meaning which feature has a small size or is "nano", will be qualified as nanocoating. Besides, nanolayers or nanostructures that exceed nanometers' dimensions may build coating while keeping nano-properties. The nanoparticles' minuscule sizes allow for more surface area per unit mass, which improves reactivity and capacity depending on the application. The product may be engineered to have improved surface area while reducing coating costs. Traditional coatings have some drawbacks, such as incorrect adhesion of the coating layer to the substrate, reduced flexibility, weakness, durability, and poor adhesion to nonporous substrates. This problem of traditional coating solutions can be solved by understanding the properties of nanocoating. Nanocoating are commercially 
available in various areas such as Anti-fingerprint, antimicrobial, self-cleaning, abrasion \& wear-resistant, thermal barrier, flame retardant, anti-reflective, self-healing, antifouling, anti-graffiti [3].

Nanomaterials are a significant part of developing human lifestyles. A new science and technology field, called "nanoscience and nanotechnology", was born with nanomaterials' development. The development of nanotechnology is mainly because of the peculiar characteristics of the materials applied in complex science and technology. Today the major global threats can be classified into two categories-energy and environment cleanliness [4]. As shown in Fig. 1, these major nanomaterials exist in organic, inorganic and hybrid nanomaterial types.

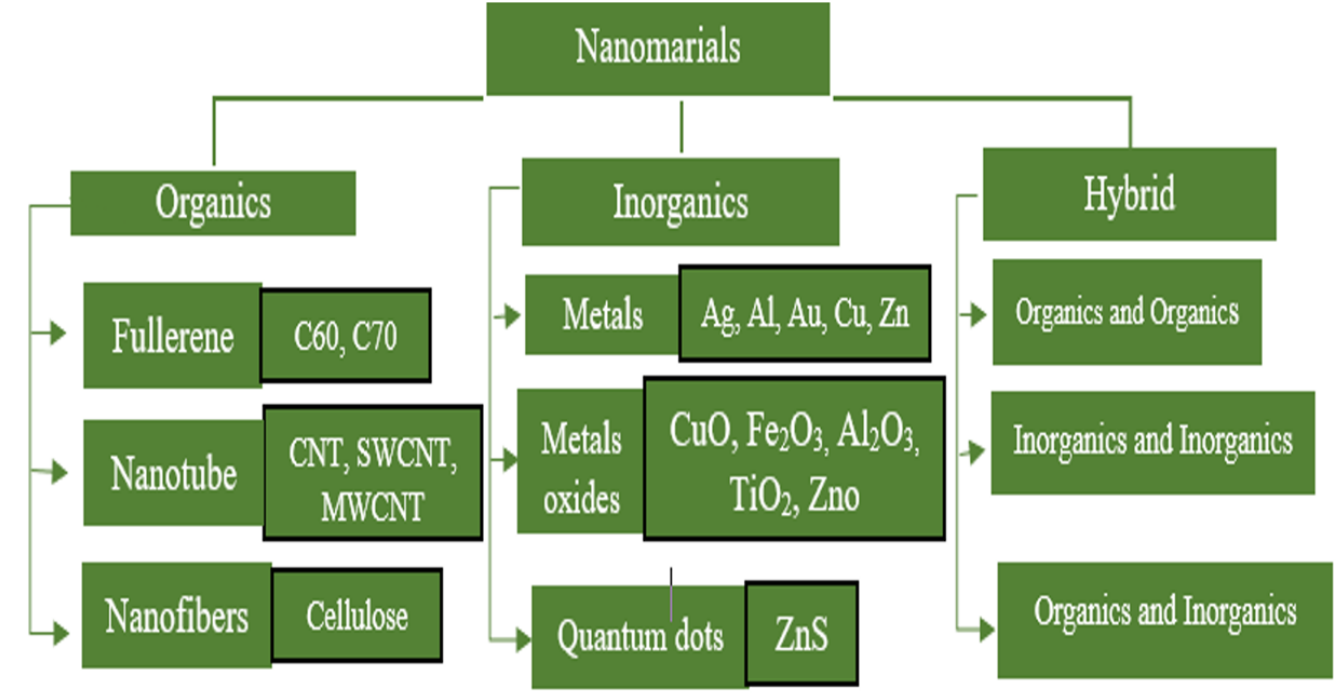

Fig. 1. Types of nanoparticles.

\section{ANTI-REFLECTIVE NANOCOATING}

\section{A. Concept of Anti-Reflection}

Reflecting is an optical phenomenon when the light is passing through a medium such as water or glass. It suddenly changes direction such that light appears to go in a new direction. An optical parameter called refractive index defines a transparent medium's optical properties, which specifies light speed in the medium versus the vacuum's speed in the vacuum. Fresnel-reflection loss is developed when an optical interference is caused within the glass by a sudden refraction index change. It is an optical phenomenon utilized to decrease the difference between the incoming and outgoing beams and generate a clear image. It can be represented as a function of incoming light intensity. The fragment of rebound light rays is defined as reflectance (R), while the rest of the transferred light is known as transmission. Moreover, The introductory guide to indicate the extent of light transmission via the medium is the refractive index .
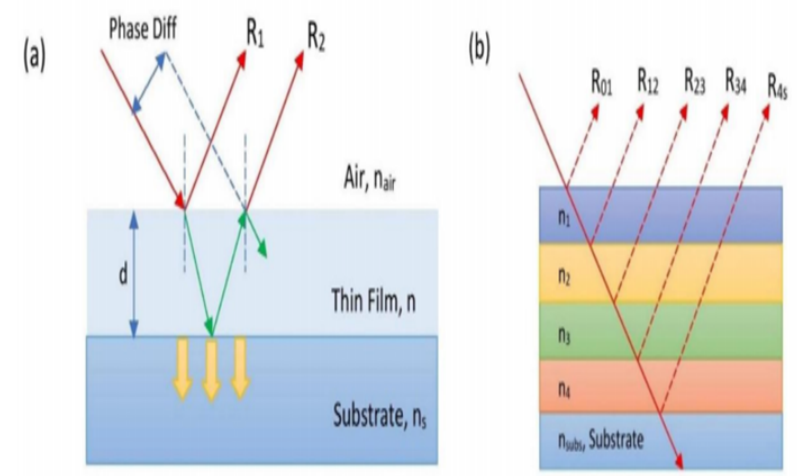

Fig. 2. Diffusion of light rays. (a) A single layer film on a substrate (ns > n). (b) Multiple layer film [5].
A film with a low index of refraction (n), which is less than the substrate, ns (i.e., $\mathrm{n}<\mathrm{ns}$ ) be the principle behind the antireflective coating. There are two interfac+es between these thin-film configurations, which cause two reflected waves, as shown in Fig. 2a. When the two wavefronts are out of phase, destroying interference occurs, completely removing both the rays and improving the transmission. The reflected waves' requirements are as follows: (i) After reflection on two interfaces, the two waves should be $180^{\circ}$ out phase and of the same intensity. (ii) The film's depth shall be one-fourth of the incident wavelength of multiple-beam numbers $(\lambda / 4)$. The reflectivity equation at normal incidence is as follows,

$$
r=\left[\frac{n_{a i r} n_{S}-n^{2}}{n_{\text {air }} n_{S}+n^{2}}\right]^{2}
$$

Only in the narrow wavelength range can the single components be used. The stacking of several thin film layers has proven to improve the coating's visual performance in a comprehensive, low-reflectivity wavelength range and various incident angles. For the multi-layered anti-reflection coatings, the principle remains the one, but in this case, the mathematical model includes analyzing individual reflected rays. As shown in Fig. 2b, light from the two layers intersection ( $\mathrm{i}$ and $\mathrm{j}$ ) is shown in the equation (if there are no losses) as follows .

$$
R_{i j}=\left|R_{i j}\right| \exp \left[-2\left(\delta_{i}+\delta_{j}\right)\right]
$$

Where $\left|R \_m n\right|=\left[\left(n \_i-n \_j\right) /\left(n \_i+n \_j\right)\right]$ and $\delta \_i=2 \pi n \_i$ di $\cos \theta_{-} \mathrm{i} / \bar{\lambda}$ (di (optical film thickness),$\theta_{-} \mathrm{j}$ (angle of refraction) and $\lambda$ (wavelength of the light)) Then, the total 
reflectivity ( $\mathrm{R}$ _sum) is obtained by integrating reflectance at the interface of each layer, which is expressed as [5]:

$$
\begin{aligned}
& R_{\text {sum }}=R_{01}+R_{12}+R_{23}+\cdots+R_{n s} \\
& R_{\text {sum }}=R_{01}+R_{12}+R_{23}+R_{4 s} \\
& \text { where } R_{01}=\left|R_{01}\right| \\
& R_{12}=\left|R_{12}\right| \exp \left[-2\left(\delta_{1}\right)\right] \\
& R_{23}=\left|R_{23}\right| \exp \left[-2\left(\delta_{1}+\delta_{2}\right)\right] \\
& R_{34}=\left|R_{34}\right| \exp \left[-2\left(\delta_{1}+\delta_{2}+\delta_{3}\right)\right] \\
& R_{4 s}=\left|R_{4 s}\right| \exp \left[-2\left(\delta_{1}+\delta_{2}+\delta_{3}+\delta_{4}\right)\right]
\end{aligned}
$$

Thus, the R_sum It can be reduced appropriately to produce an anti-reflection effect by optimizing refracting and optical film thickness index.

\section{B. Criteria for Best Anti-Reflection Coating}

Broadband anti-reflectivity: The ARC should have a suitable wavelength of the AR properties range. ARCs should cover a wide range, including an Ultraviolet (UV) area or an infrared area.

Omnidirectional anti-reflectivity: The refractive index of glass or plastic products is about 1.5 and a reflection of $4 \%$ at normal incidence but reach up to $100 \%$ at grazing angles. In the same context, anti-reflection coatings are also presented with such a phenomenon, and many coatings produce excellent anti-reflection characteristics, mainly from 30 to 60 within an incident. Therefore Omni-directional reflectivity is a crucial feature to be optimized.

High stability: The ARCs should be well connected to the surface and must be inert with excellent stability in their operating environment.

Multifunctionality: ARCs in the solar collector and PV cell mainly aid in reducing the loss of reflection. Conversely, hydrophobic self-cleaning properties can also help solve the dust problem [6].

Cost: The low production costs make it more attractive, apart from all the properties desired.

\section{Anti-Reflection Coating Materials}

Anti-reflective coating's main task is to reduce reflective loss and assist the maximum transmission of light into a glass. Criteria, where ARC's material determines the coating revolution's efficacy, the ARC structure (primarily the top surface), and the manufacturing methods fabricating, prepare an Anti-reflection coating. Materials, including transparency, weight, mechanical robustness, thermal, corrosion, and chemical inertia, will play an important role. Furthermore, the manufacturing method highly depends on material properties and influences the factor of costs [7]. The ARC material classification is shown in the following Fig. 3.

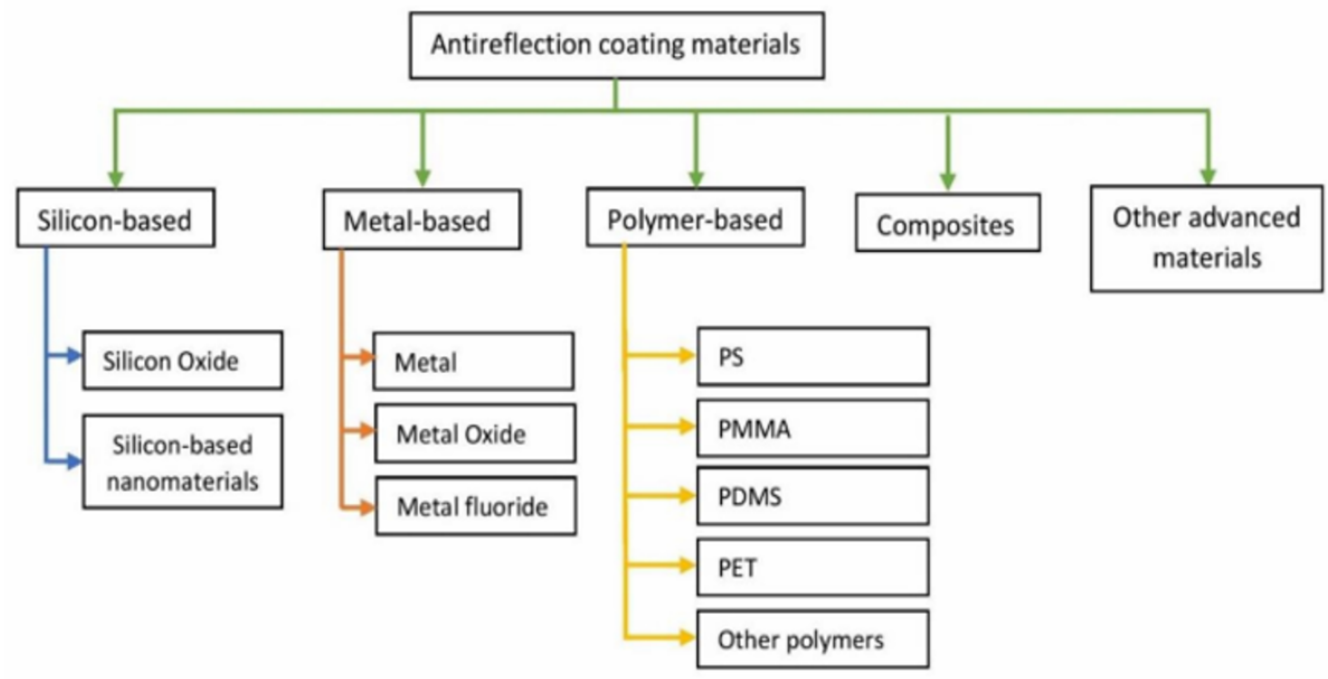

Fig. 3. Anti-reflective materials classification [8].

\section{Fabrication Method}

The manufacturing methods are the key steps where various parameters of manufacture can modify the required features. For a specific application, the preparation method's choice is essential after selecting the AR coating material. Manufacturing precise constructions, more straightforward control, flexibility and cost-effective methodology are some significant results. Generally, the manufacturing techniques are classified under conventional techniques as a top-down and a bottom-up approach [9]. The majority of nanostructurebased ARCs may use the same comprehensive subtractive top-down or additive bottom-up nanotechnology. Some unconventional manufacturing techniques are often used for anti-reflection coatings. The classification of ARC manufacturing methods is shown in Fig. 4.

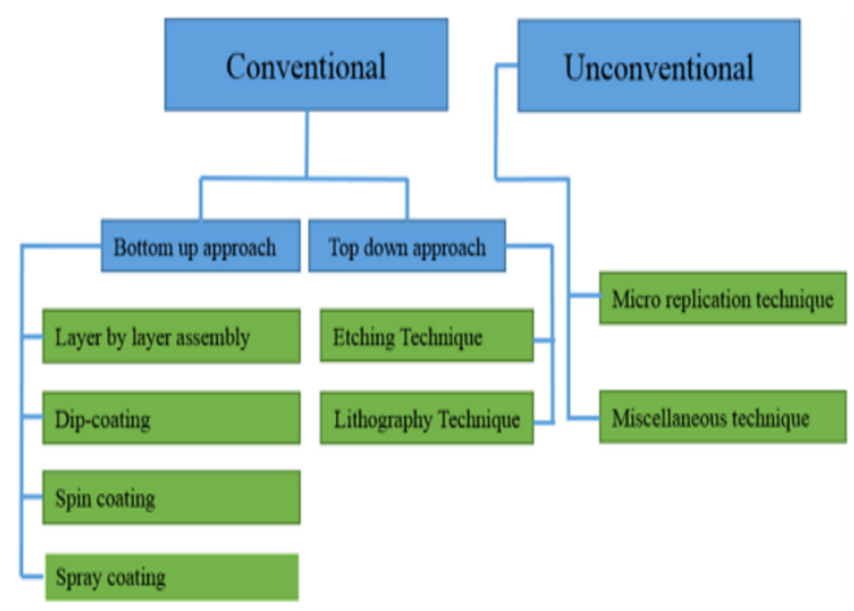

Fig. 4. Manufacturing methods for anti-reflective coating [10]. 


\section{Self-Cleaning Nanocoating}

Glass is used in various fields, such as industry, building, construction, and solar energy. It is also used in places that are very hard to reach in buildings every day. Therefore, nanotechnology has given create a new kind of self-cleaning glass (SCG). In SCG, the bottom-up or top-down approach creates a thin layer with silica or titanium on the glazing surface of approximately $1025 \mathrm{~nm}$. Control of the surface wettability results in self-cleaning. New interfaces between different materials are created by surface coated on solid substrates. Consideration should be given to wearability, interface evolution, adhesion properties, and spreading. A solid's wettability with a liquid is defined by the substance's contact angle [11]. The angle of contact $(\theta)$, as illustrated in Fig. 5, is obtained through a balance of interfacial tensions and described in Youngs' equation.

$$
\sigma_{s v}=\sigma_{s 1}+\sigma_{1 v} \cos \theta
$$

where $\left(\sigma_{s v}, \sigma_{s 1}\right.$ and $\left.\sigma_{1 v}\right)$ are the interfacial energies of liquidvapour, solid -vapour and solid-liquid interfaces per unit area.

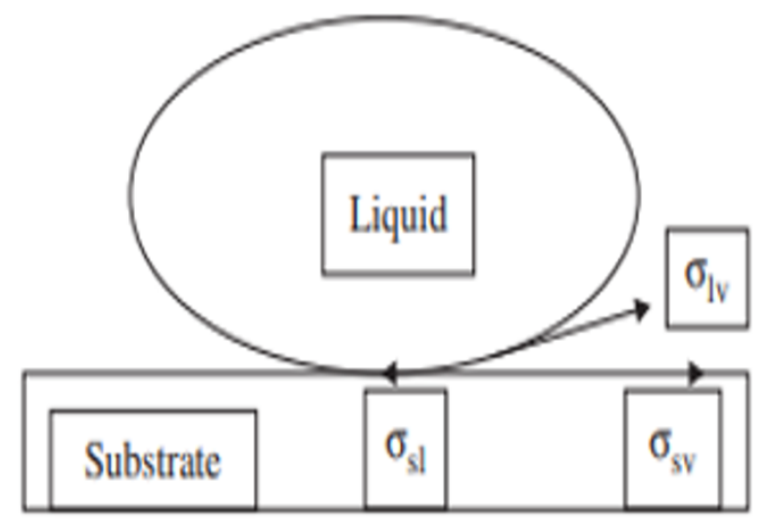

Fig. 5. Interfacial tensions on the solid substrate

The contact angle $(\theta)$ on a solid surface of the liquid droplet has described the angle between a solid surface and the fluid droplet's tangent, as illustrated in Fig. 6. $(\theta)$ generally used to determine the wettability of the surface.
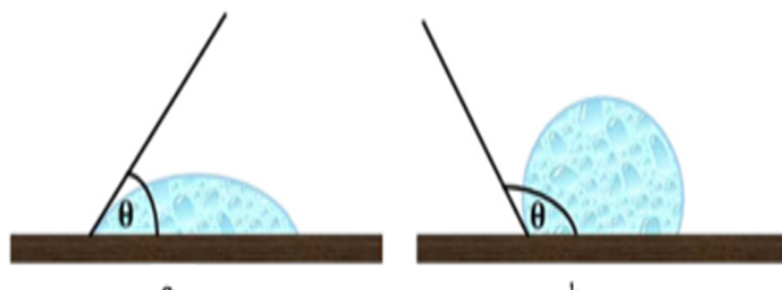

Fig. 6. Schematic illustration of a liquid drop and contact angle. (a) Hydrophilic surface. (b) Hydrophobic surface [12].

Self-cleaning surfaces can be used in a solar system because they are accessible to dust removal from the surface to enhance overall energy conversion and light transmission efficiency. The surfaces are also classified into two categories: (a) surface hydrophilic and (b) hydrophobic surfaces. Water drops transmitted throughout the surface and forms a hydrophilic water film. The water drains roll out rapidly from the surface on hydrophobic surfaces by waterresistant and low hydrophobic surface mechanical characteristics and eliminate the pollutants from the surface. Fig. $7 \mathrm{a}$ and $7 \mathrm{~b}$ described the above two pictorially

Mechanisms for SC.

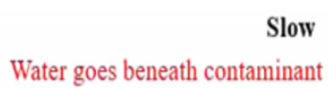

(a)

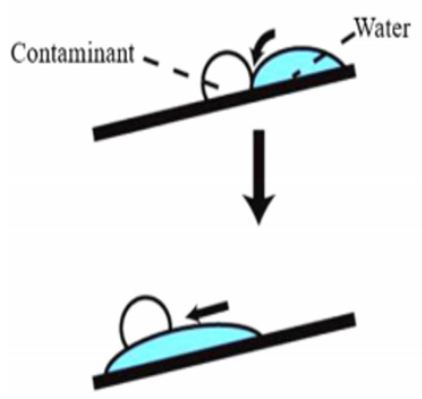

Fig. 7. Schematic of self-cleaning processes (a) Superhydrophilic surface. (b) Superhydrophobic surface [12].

As previously mentioned, a few species of plants had also developed superhydrophobic "auto-cleaning", which absorbs water for highly efficient. The most popular among them is the lotus leaf. Its remarkable survival and adaptation strategies enable its leaves to be kept clean up a droplet and remove contaminants keep leaves clean. A higher water contact angle $\left(\mathrm{CA}>150^{\circ}\right)$, known as super hydrophilicity, is where droplets smoothly rolled off the surface under $10^{\circ}$ at a sliding angle to remove contaminants from the surface. Lotus Effect is famous and occurs in the leaves of the plant. The ruggedness of the lotus leaf has been proposed mainly to account for its non-wetting properties. Epical wax and hierarchy with micro and nanostructures are the significant drivers of lotus leaves' super hydrophilicity [13]. Surfaces are marked by their contact angle (CA), as shown in Table I.

TABLE I: CONTACT ANGLE (CA) VALUES AND WETTABILITY BEHAVIOR [14]

\begin{tabular}{cc}
\hline \hline Contact angle $\left({ }^{\circ}\right)$ & Wettability \\
\hline \hline$\theta>90^{\circ}$ & Hydrophobic \\
$\theta>150^{\circ}$ & Superhydrophobic \\
$0<\theta<90^{\circ}$ & Hydrophilic \\
$\theta<10^{\circ}$ & Superhydrophilic \\
\hline \hline
\end{tabular}

\section{Nanocoating Of Glass as A Technology for SOLAR APPLICATIONS}

The schedule shown in Fig. 9 shows an understanding of glass technology and glass coating for solar energy. At the beginning of 1960, float glass was finally introduced into the architecture industry. Float glass became immensely important as very high-quality material. The information about the glass processes is well developed and has been extended by various researchers over the years. FPSC were manufactured for water-heating applications with the initiation of the energy crisis that began in 1970, marking this material's creation as a technology used in this field. Indeed, the development of the distributed harvesting of solar energy was a big step in developing energy. In 1980, the composition was modified, and the light transmission improved within 900-1100 nm. The extensive deposition of transparent conducting oxides and semiconductor materials and their modelling contributed to the development and progress in solar energy use. Hence, the energy output supply also increased. 


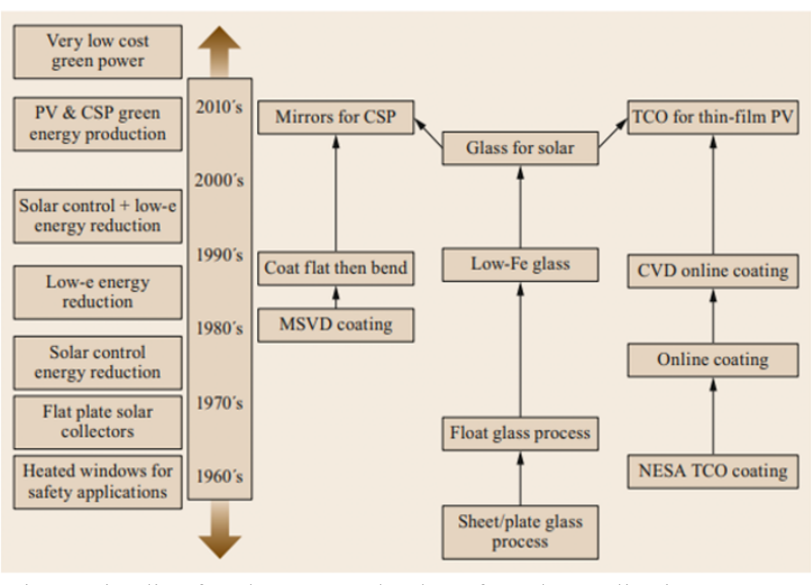

Fig. 8. Timeline for glass as a technology for solar applications [15].

The glass surface can be made through a float process that brings a strong concept for coatings' deposition, enhancing optical performance or durability. Glass has been confirmed as the preferred material for solar thermal collectors and concentrated solar power (CSP) and PV applications with all these characteristics and its ability to change them with the control of the composition of glass. It is also essential for ensuring the chemical and mechanical durability required to live years outdoors. This work presents the literature of the experimental and theoretical studies of anti-reflection and self-cleaning coating on FPSC cover glass.

Reference [16] evaluated the particular kind of coating using a basic sol-gel prosses called superhydrophobic solar selectively. With $89.46 \%$ solar absorption without corrosion, a Selective glass absorber can withstand conditions outside. The borosilicate glass selective absorber can provide more extraordinary performance.

Reference [17] examined the materials of glass and heat removal factor. The glass material of polyvinyl fluoride provides more transmissibility than the other material. The higher heat removal factor coefficient of heat loss has a significant impact on the collector's performance.

Reference [18] prepared a glass substrate and created a multilayer by DC-reactive magnetron sputtering with $\mathrm{Cu} 2 \mathrm{O}$, nanocomposite $(\mathrm{Cu} 2 \mathrm{O}$ and $\mathrm{Ag})$ and multilayered thin films ( $\mathrm{Cu} 2 \mathrm{O}$ and $\mathrm{Ag} 2 \mathrm{O}$ ). The coupling of $\mathrm{Ag} 2 \mathrm{O}$ with $\mathrm{Cu} 2 \mathrm{O}$ was found to improve the absorption of light.

Reference [19] developed a special coating designed of transparent conductive oxides with high transmittance and low emissivity. This coating improves the thermal performance of single and double glass collectors.

Reference [20] used the porous gel coating on the collector glass cover, which transmits radiation better than other coatings. The valuable collector energy is increased by 1.07 and 1.04, respectively, at small and high incident angles.

Reference [21] measured the double-glazed effect in the system's performance and compared it with a single-glazed collector. For a double-glass and single-glass, collection effectiveness was $55 \%$ and $12 \%$, with the same conditions as the intensity and dimensions of solar radiation, respectively.

Reference [22] studied optical properties of two different types of dielectric materials, namely titanium dioxide (TiO2) and silicon dioxide ( $\mathrm{SiO} 2)$ deposited by a sputtering method, to create anti-reflective coatings for solar applications. The spectroscopic result showed that the sputtering process could successfully be used in solar thermal applications due to the low absorbing and reflective efficiency of up to $70.0 \%$, the high solar transmission of about $87 \%$ for bilayer films $70 \%$ for the three-layer film.

Reference [23] reported the results to show that the annual energy output at a solar heating plant, which has $100 \mathrm{C}$ solar fluid collector temperature, can be increased by approximately $20 \%$ with ARC on the sunglass cover using silicon diodes as an ARC on the solar thermal collector.

\section{CONCLUSION}

This study aims to present the review and analysis of the recent development of performance enhancement methodologies for FPSC in various applications. The article review shows that nanotechnologies can play a crucial role in meeting requirements by improving the primary properties of materials and adding new functionalities to existing material and products, as illustrated by self-cleaning and antireflective. Nanotechnology also can play an essential role in improving solar collectors' performance. This review provided information on the work of nanocoating in solar collector system. Nanocoating there is great potential to improve the solar thermal collector's efficiency by covering nanoparticles to increase the glass cover's optical characteristics. Today, all the nanomaterials research has improved optical properties, but some aspects like the thermal stability in the high-temperature field require further improvements. The size of nanoparticles also has an impact on the solar collector's efficiency. As mentioned above in the study, this work will focus more on reducing collector heat loss and increasing the solar flat plate collector's optical and thermal performance using nanofluid as working fluid and nanocoating (anti-reflective and self-cleaning) on the glass cover.

\section{ACKNOWLEDGMENT}

This work was supported by the Stipendium Hungarian Program and by the Doctoral School of Mechanical Engineering, Institute of Technology, the Hungarian University of Agriculture and Life Sciences, Gödöllö, Hungary.

\section{CONFLICT OF INTEREST}

Authors declare that they do not have any conflict of interest.

\section{REFERENCES}

[1] Ullah KR, Saidur R, Ping HW, Akikur RK, Shuvo NH. A review of solar thermal refrigeration and cooling methods. Renewable and Sustainable Energy Reviews. 2013; 24: 499-513.

[2] Serrano E, Rus G, García-Martínez J. Nanotechnology for sustainable energy. Renewable and Sustainable Energy Reviews. 2009; 13(9): 2373-2384.

[3] Aravind S, Prabhu MG, Kaushik D, Kumar KLS. Review on Nanocoating for Bio-Implants. International Research Journal of Engineering and Technology. 2017; 4(10): 682-686.

[4] Petkoska-Trajkovska A, Nasov I, Jovanovski L. Nanocoatings: 
Designed layers for solar thermal applications. Zastita Materijala 2016; 57(1): 5-12.

[5] Raut HK, Ganesh VA, Nair AS, Ramakrishna S. Anti-reflective coatings: A critical, in-depth review. Energy and Environmental Science. 2011; 4(10): 3779-3804.

[6] Reddy KS, Kamnapure NR, Srivastava S. Nanofluid and nanocomposite applications in solar energy conversion systems for performance enhancement: A review. International Journal of LowCarbon Technologies. 2017; 12(1): 1-23.

[7] Yao L, He J. Recent progress in antireflection and self-cleaning technology - From surface engineering to functional surfaces. Progress in Materials Science. 2014; 61: 94-143.

[8] Shanmugam N, Pugazhendhi R, Elavarasan RM, Kasiviswanathan P, Das N. Anti-reflective coating materials: A holistic review from PV perspective. Energies. 2020; 12(10): 1-93.

[9] Han Z, Jiao Z, Niu S, Ren L. Ascendant bioinspired antireflective materials: Opportunities and challenges coexist. Progress in Materials Science. 2019; 103: 1-68.

[10] Hanaei H, Assadi MK, Saidur R. Highly efficient antireflective and self-cleaning coatings that incorporate carbon nanotubes (CNTs) into solar cells: A review. Renewable and Sustainable Energy Reviews. 2016; 59: 620-635.

[11] Ji S, Song K, Nguyen TB, Kim N, Lim H. Optimal moth eye nanostructure array on transparent glass towards broadband antireflection. ACS Applied Materials and Interfaces. 2013; 5(21): 10731-10737.

[12] Banerjee S, Dionysiou DD, Pillai SC. Self-cleaning applications of $\mathrm{TiO} 2$ by photo-induced hydrophilicity and photocatalysis. Applied Catalysis B: Environmental. 2015; 176-177: 396-428.

[13] Mehmood U, Al-Sulaiman FA, Yilbas BS, Salhi B, Ahmed SHA, Hossain MK. Superhydrophobic surfaces with antireflection properties for solar applications: A critical review. Solar Energy Materials and Solar Cells. 2016; 157: 604-623.

[14] Zhang L, Dillert R, Bahnemann D, Vormoor M. Photo-induced hydrophilicity and self-cleaning: Models and reality. Energy and Environmental Science. 2012; 5(6): 7491-7507.

[15] Viallet V, Seznec V, Hayashi A, Tatsumisago M, Pradel A. Glasses and Glass-Ceramics for Solid-State Battery Applications. 2019.

[16] Zhu Y, Shi J, Huang Q, Fang Y, Wang L, Xu G. A superhydrophobic solar selective absorber used in a flat plate solar collector. RSC Advances. 2017; 7(54): 34125-34130.

[17] Dondapati RS, Agarwal R, Saini V, Vyas G, Thakur J. Effect of Glazing Materials on the Performance of Solar Flat Plate Collectors for Water Heating Applications. Materials Today: Proceedings. 2018; 5(14): 27680-27689.

[18] Tseng CC, Hsieh JH, Wu W. Microstructural analysis and optoelectrical properties of $\mathrm{Cu}_{2} \mathrm{O}, \mathrm{Cu}_{2} \mathrm{O}-\mathrm{Ag}$, and $\mathrm{Cu}_{2} \mathrm{O} / \mathrm{Ag}_{2} \mathrm{O}$ multilayered nanocomposite thin films. Thin Solid Films. 2011; 519(15): 5169-5173.

[19] Giovannetti F, Föste S, Ehrmann N, Rockendorf G. High transmittance, low emissivity glass covers for flat plate collectors: Applications and performance. Solar Energy. 2014; 104: 52-59.

[20] Khoukhi M, Maruyama S, Komiya A, Behnia M. Flat-plate solar collector performance with coated and uncoated glass cover. Heat Transfer Engineering. 2006; 27(1): 46-53.

[21] Vettrivel H, Mathiazhagan P. Comparison study of solar flat plate collector with single and double glazing systems. International Journal of Renewable Energy Research. 2017; 7(1): 267-274.

[22] Boudaden J, Ho RSC, Oelhafen P, Schüler A, Roecker C, Scartezzini JL. Towards coloured glazed thermal solar collectors. Solar Energy Materials and Solar Cells. 2004; 84(4): 225-239.

[23] Furbo S, Jivan Shah L. Thermal advantages for solar heating systems with a glass cover with antireflection surfaces. Solar Energy. 2003; 74(6): 513-523. 